\title{
MOLECULAR BIOLOGICAL ENHANCEMENT OF COAL BIODESULFURIZATION
}

\section{SEVENTH QUARTER REPORT (May-July 1990)}

Prepared by

John J. Kilbane

Barbara A. Bielaga

IGT Project No. 61095

for

U.S. Department of Energy

Pittsburgh Energy Technology Center

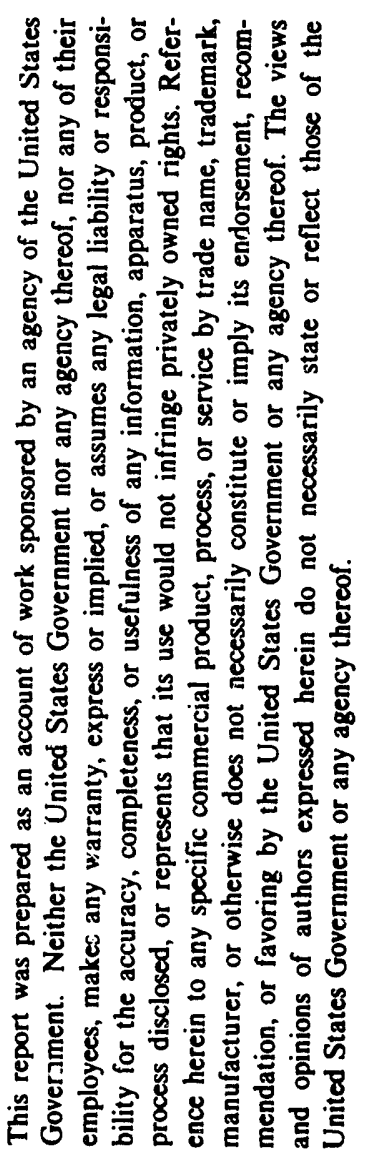

DOE Contract No. DE-AC22-88PC8891

Date Published - July 1990

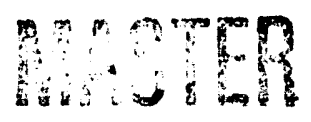


EXECUTIVE SUMMARY

During chis quarter (May to July 1990) preliminary data were obtained concerning the kinetics of coal biodesulfurization, genetic experiments led to the development/investigation of new methodologie.., and a new assay for desulfurization based on the metabolism of met: ylene blue dye was evaluated.

Experiments of 2 to 6 weeks duration were performed in which coal served as the only source of sulfur for the growth of microbial cultures. The results of ASTM analyses indicate as much 35478 reduction in organic sulfur; however, elemental analyses were also performed on these coal samples and the ratios of aluminum-to-sulfur or silicon-to-sulfur do not indicate significant reductions of sulfur. These experiments are on-going and the analysis of these samples by other techniques, as well as the analysis of additional samples, will hopefully resolve these inconsistancies.

Genetic experiments involving electroporation hav's led to the development of a simplified method that can yield a high number of transformants in a fraction of the time required to perform previous procedures. A preprint of a manuscript describing the new procedure is appended to this report. In other genetic studies, the lack of a cloning vector that replicates in $\underline{R}$. rhodochrous has limited the scope of genetic experiments that can be performed with IGTS8. To overcome this limitation, a new approach has been taken. Segments of the IGTS8 chromosome have been cloned into the E. coli vector pBR325. Such plasmids are unable to replicate in $\underline{R}$. rhodochrous, but through homologous recombination should be able to integrate into the chromosome of IGTS8. Rare transformants have been obtained using this technique, and DNA hybridization experiments will be performed to confirm the identity of these presumptive transformants. This technique should allow the optimum conditions for the transformation/electroforation of IGTS8 to be determined even though a cloning vector for IGTS8 is not yet available.

The screening of large numbers of colonies for the presence or absence of desulfurization ability is an integral part of this project. Currently, the most convenient screening assay is the Sulfur Bioavailability Assay; however, a more convenient assay based on the metabolism of the dye methylene blue, which happens to be an organosulfur compound, has been investigated. IGTS8 decolorizes methylene blue, whereas other strains of $\underline{R}$. rhodochrous do not; 
however, the decolorization of methylene blue, as a result of the specific cleavage of carbon-sulfur bonds, has not yet been demonstrated.

During the next quarter, coal biodesulfurization experiments will continue, genetic studies will focus on utilizing and developing techniques for the genetic analysis of $\underline{R}$. rhodochrous, and the validity of a desulfurization assay based on methylene blue dye will be established. 
TABLE OF CONTENTS

\section{Page}

OVERALL OBJECTIVE

1

WORK PLANNED FOR THE CURRENT QUARTER

2

WORK PERFORMED DURING THE CURRENT QUARTER

Experiments With Coal

3

Development of Genetic Techniques

5

Development of a New Desulfurization Assay

6

WORK PLANNED FOR THE NEXT QUARTER

GLOSSARY

APPENDIX. Insiantaneous Gene Transfer From Donor to Recipient Microorganisms Via Electroporation

$A-1$ 


\section{LIST OF FIGURES}

Figure No.

page

1

Spectrogram of 0.058 Methylene Blue Control

7

2

Spectrogram of IGTS 8 Cell Ccntrol

8

3

Spectrogram of 0.058 Methylene Blue Plus IGrs 8 Cells

9

LIST OF TABLES

Table No.

Page

1

Coal Biodesulfurization Kinetic Studies

4 
OVERALL OBJECTIVE

The overall objective of this project is to use molecular genetics to develop strains of bacteria with enhanced ability to remove sulfur from coal and to obtain data that will allow the performance and economics of a coal biodesulfurization process to be predicted. 
WORK PLANNED FOR THE CURRENT QUARTER

The work planned for the current quarter (May 1990 to July 1990) includes

the following activities:

- Construct a cloning vector that can be used in Rhodococcus rhodochrous IGTS8 from the small cryptic plasmid found in Rhodococcus rhodochrous ATCC 19067

- Develop techniques for the genetic analysis of IGTS8

- Continue biochemical experiments, particularly those that may allow the identification of desulfurization-related enzymes

- Continue experiments with coal to determine the kinetics of organic sulfur removal. 
WORK PERFORMED DURING THE CURRENT QUARTER

\section{Experiments With Coa?}

Studies performed prior to this project indicated that significant coal organic sulfur reductions can be obtained using very long microbial treatment times. Experiments to investigate the kinetics of organic sulfur removal are currently underway and preliminary results are reported here. For this test work, the mixed microbial culture IGTS7, as weil as the pure Rhodococcus rlodochrous culture IGTS8, were included in growth experiments using the IBC-101 coal sample obtained from Dave Boron of the U.S. DOE/PETC. This coal, which contained an organic sulfur content of 2.878 and a total sulfur content of 3.168 , was used in experiments that contained approximately 1 gram of coal in a total volume of 2 liters. After incubation, the coal was recovered by centrifugation, washed free of bacteria, and submitted for analysis. Analysis included sulfur forms, ash, aluminum, and silica in ash, proximate, and ultimate. Mass balance determinations were also recorded. These results are summarized in Table 1.

The data for organic sulfur (as determined by the ASTM procedure) suggest that sulfur reductions up to 478 may have been achieved; however, the elemental ratios ( $\mathrm{Al} / \mathrm{S}$ and $\mathrm{Si} / \mathrm{S}$ ) do not support this observation. Moreover, those samp ${ }^{2}=$ that appear to have experienced the highest removals of organic sulfur also experienced coal recovery weights in excess of the amount of coal added. This suggests that incomplete sample washing occurred with these samples, yielding a mixture of biomass as well as coal. Sample Nos. 1, 3, and 7 listed in Table 1 consisted of mixtures of appreciable quantities of biomass and coal; whereas, the remaining samples consisted only of coal, which should be reflected by the elemental ratios and the ash content values. The data are inconclusive, but without further analyses it is not possible to conclude that organic sulfur removal has been achieved in these samples. These experiments are ongoing, and the further analyses of these samples and the data that will be obtained from additional coal samples that have been subjected to biotreatment for longer periods of time will hopefully resolve the inconclusive data obtained thus far. 


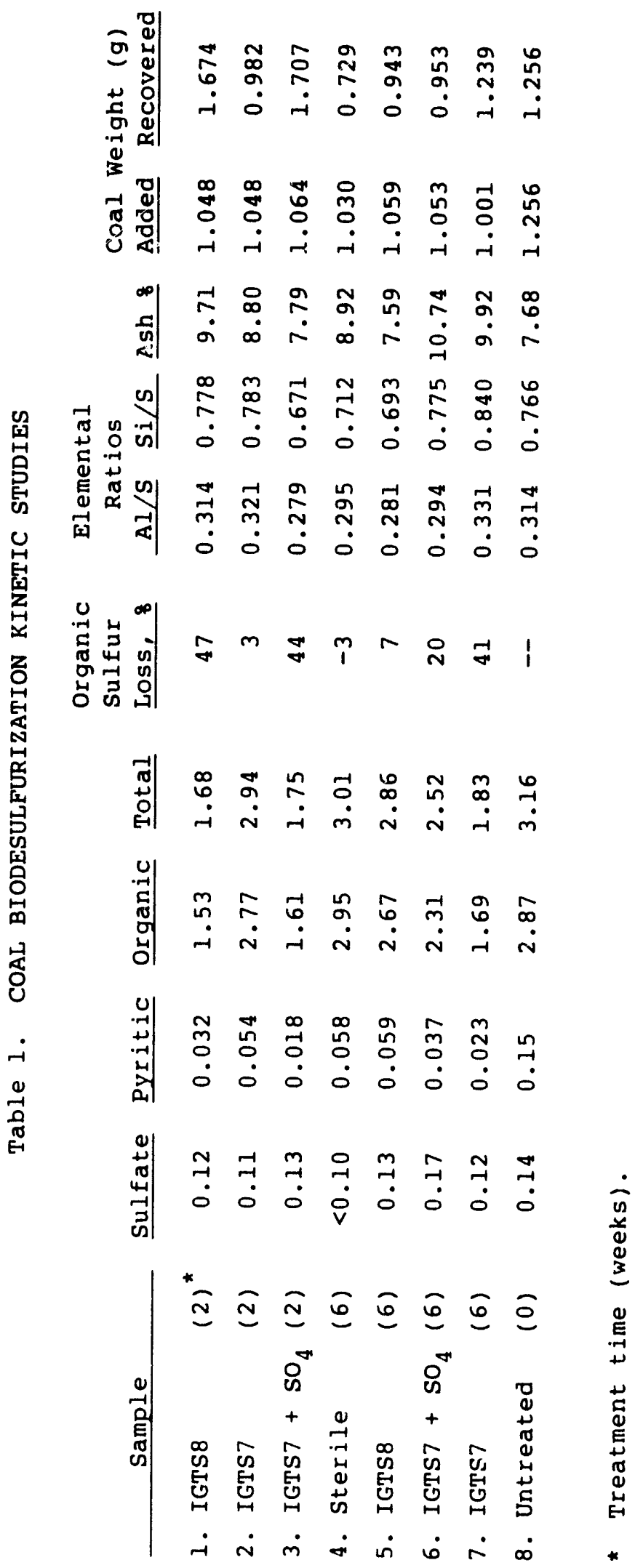




\section{Development of Genetic Techniques}

The focus of genetic experiments has been to obtain/construct a cloning vector that can be used in $\underline{R}$. rhodochrous, and with attempts to introduce genetic information into $\underline{\mathrm{R}}$. rhodochrous by transformation. The construction of a hybrid replicon by combining pBR325 with a 9 kilobasepair plasmid, pKGBl, from the ATCC $19067 \underline{R}$. rhodochrous strain has not yet been successful. The chief difficulty is obtaining purified pKGBI DNA. The ATCC 19067 does not routinely yield DNA preparations that contain plasmid DNA, and since the plasmid is cryptic we have no means by which to select for the presence of the plasmid. Experiments to achieve this goal are continuing. Additionally, a new strategy is being tried to develop a technique to introduce DNA into $\underline{R}$. rhodochrous. We have previously constructed genomic libraries of IGTS 8 by inserting chromosomal fragments into E. coli plasmids such as pBR325 and pCP13. These plasmids do not replicate in $\underline{\mathrm{R}}$. rhodochrous; however, the presence of chromosomal DNA inserts can permit homologous sequences present on these plasmids and the chromosome of IGTS8 cells to undergo recombination resulting in the integration of the entire plasmid into the chromosome. This is dependent upon two events: successful introduction of the plasmid into the cell and subsequent recombination of the plasmid with the chromosome at regions of homology. Consequently, the frequency of such events will be low, but preliminarv experiments appear to have worked. These results will be confirmed using hybridization (Southern blotting) experiments to demonstrate the insertion of pBR325 into the IGTS8 chromosome. Even though this technique results in a very low frequency of transformants, it will allow the conditions needed for the electropration/transformation of IGTS8 to be investigated and optimized.

An additional aspect of genetic research has involved the development of an improved methodology for the transformation of bacterial cells using electroporation. Electroporation is the use of a controlled discharge of electrical energy to facilitate the uptake of DNA by bacterial (or eukaryotic) cells. Electroporation is routinely performed in this project to introduce plasmid and chromosomal DNA into $\underline{R}$. rhodochrous IGTS8 and into Escherichia coli. The published procedures for electroporation seemed cumbersome so improvements in the procedures were investigated. These investigations resulted in the development of a highly simplified and reliable procedure, 
which is described in detail in the manuscript contained in the appendix. This improved electroporation method has been demonstrated to be useful for Pseudomonas species, as well as $\underline{E}$. coli ; however, transformants of $\underline{R}$. rhodochrous have not yet been obtained.

\section{Development of a New Desulfurization Assay}

The dye methylene blue contains carbon-sulfur bonds in a polyaromatic structure and may be a good compound to use in developing a desulfurization assay. Agar plates containing various concentratiors of methylene blue were prepared and streaked with various bacterial strains. IGTS8 causes clearing of the agar, whereas other $\underline{R}$. rhodochrous species and other bacteria, in general, do not. The metabolites of methylene biue produced by IGTS8 are being investigated by GC/MS and if the specific cleavage of carbon-sulfur bonds can be documented, the use of methylene blue appears to have great promise in terms of the development of a convenient desulfurization assay.

The decolorization of methylene blue can be caused by a variety of metabolic events and is not restricted to the cleavage of carbon-sulfur bonds. Nonetheless, a range of organosulfur compounds metabolized by IGTs8 have been examined by GC/MS and the reaction products indicate that the specific cleavage of carbon-sulfur bonds has occurred. Moreover, methylene blue serves as a sulfar source in the sulfur Bioavailability for IGTS 8 but not for other bacterial cultures. The decolorization of methylene blue by IGTS8 is; therefore, likely to be due to the cleavage of carbon-sulfur bonds. The metabolism of methylene blue by IGTS8 was investigated by UV/VIS spectrophotometry, and the results are shown in Figures 1 through 3 . Figure 1 is a spectrogram of a containing 0.058 methylene blue control and illustrates that the adsorption peak produced by methylene blue is most prominent at about 660 nanometers. Figure 2 is a control that contains IGTs 8 cells but no methylene blue. Figure 3 is the spectrogram produced by a mixture of methylene blue and IGTS8 cells. The methylene blue peak has almost completely disappeared; however, no new peak that might result from a metabolite of methylent blue is observed. GC/MS analysis of methylene blue and metabolites produced by IGTS8 is inhibited by the fact that the molecule is a water soluble salt and does not chromatograph well. Future experiments will attempt to derivatize the molecules prior to GC/MS or will employ analogues of methylene blue that are amenable to chromatography. 


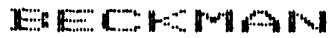

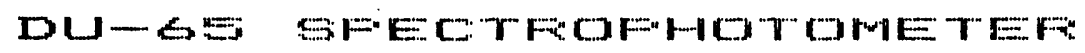

AESOFEAANCE:

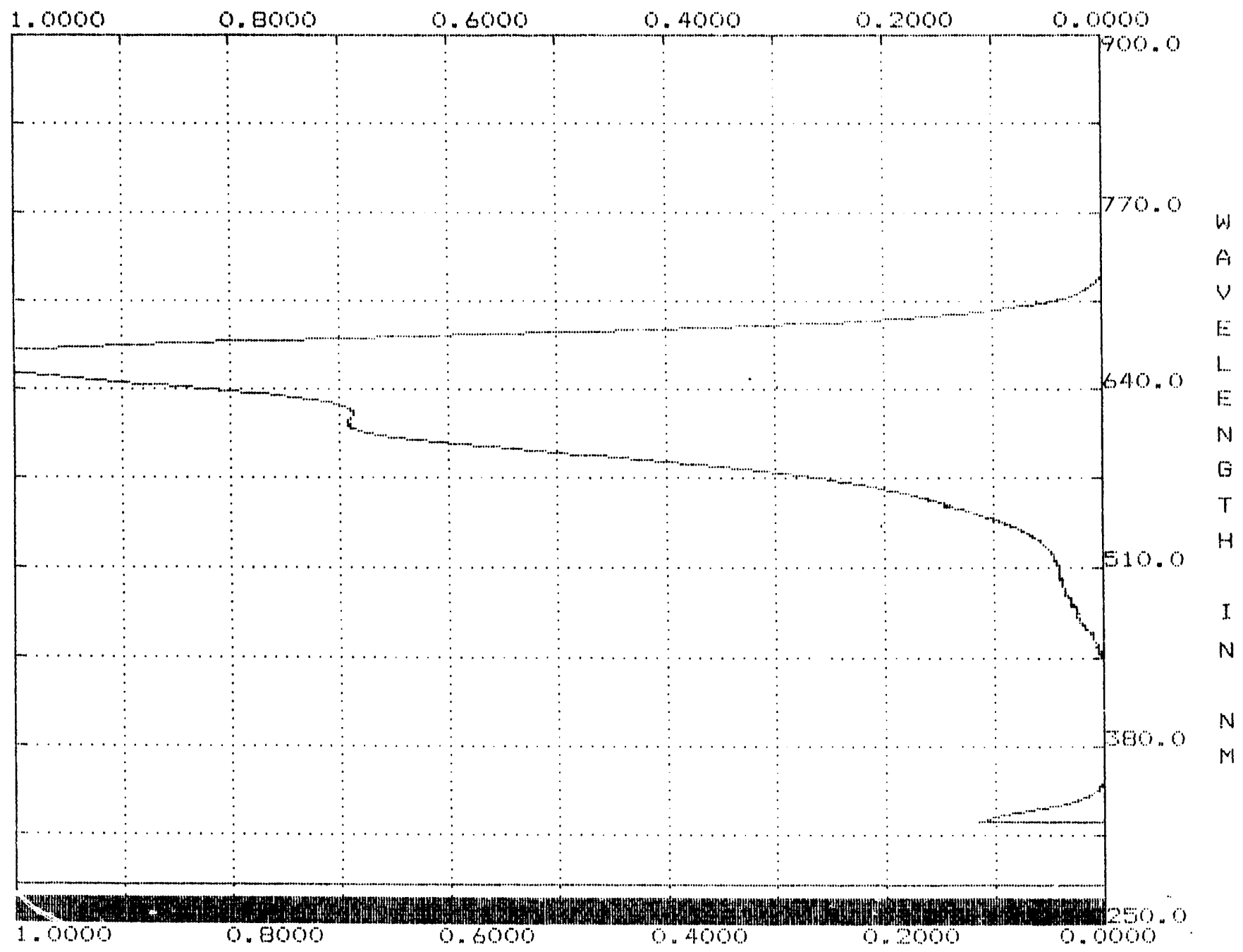

Som speed: 500 nm/mim

Figure 1. SPECTROGRAM OF 0.058 METHYLENE BLUE CONTROL 


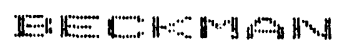

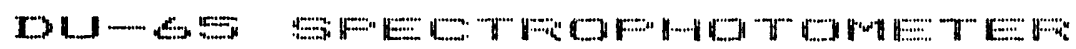

ABSOFEANAE:

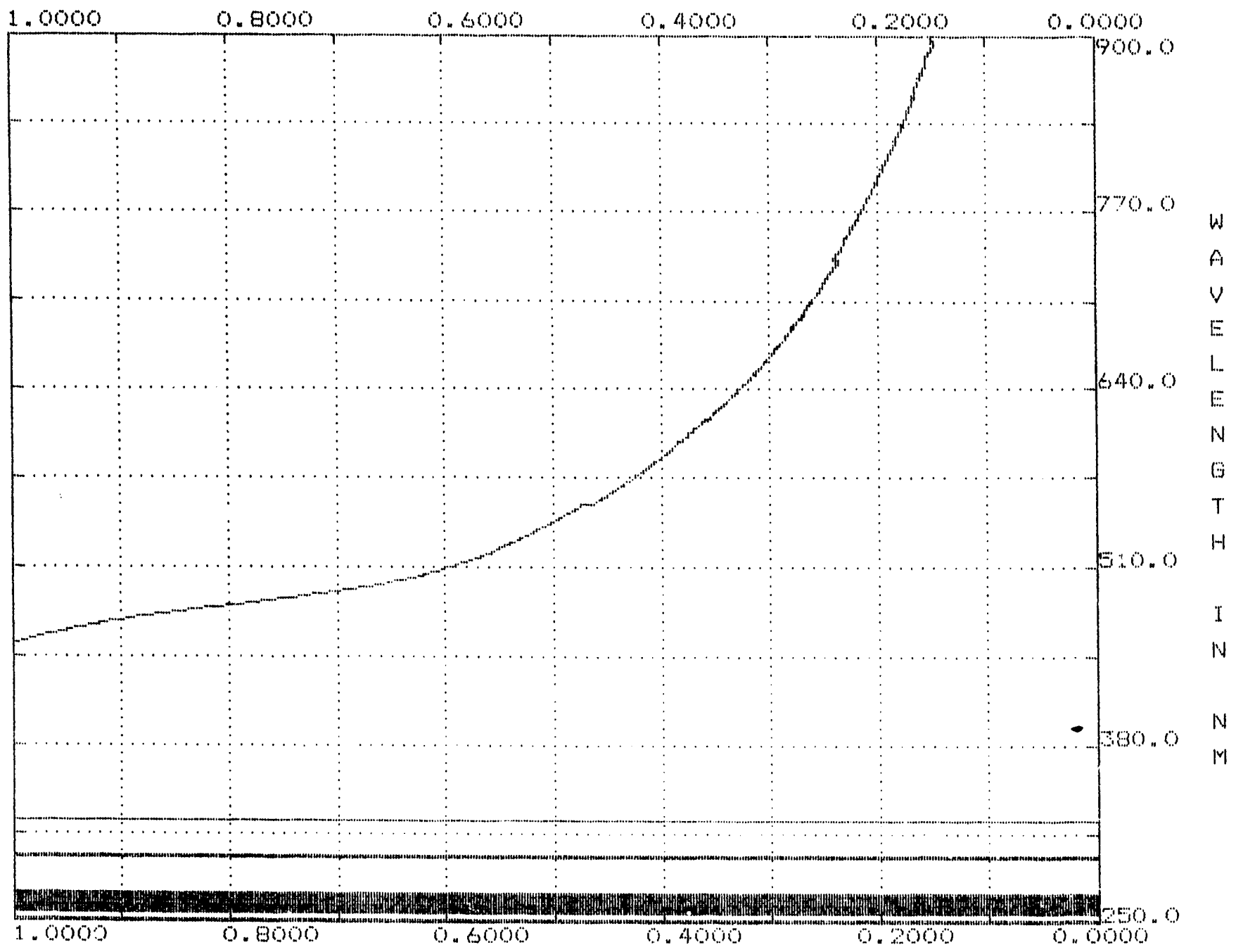

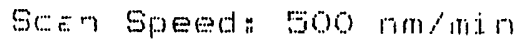

?igure 2. SPECTROGRAM OF IGTS8 CELL CONTROL 


\section{Exi}

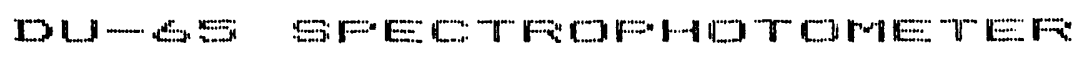

AESOREANCE

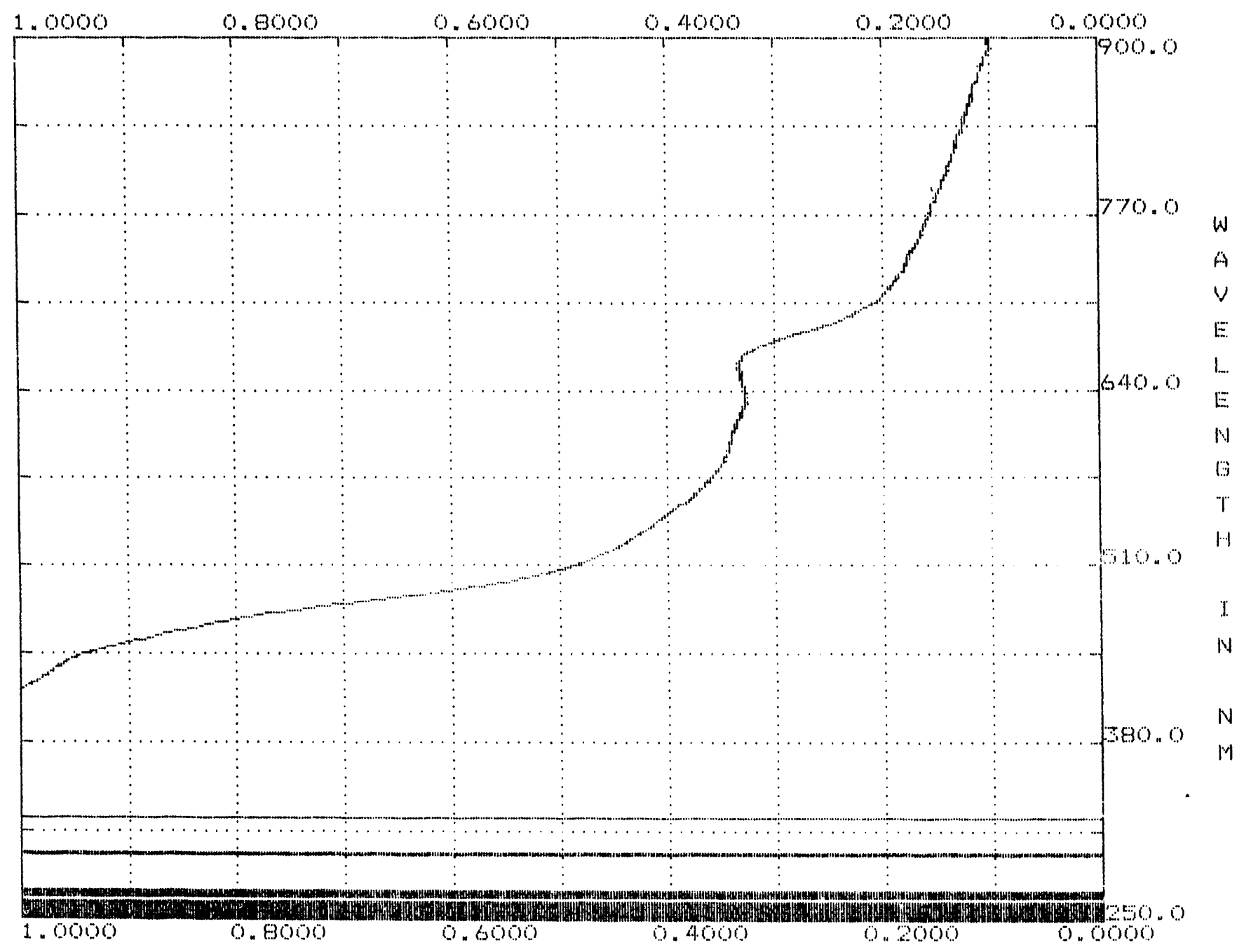

Scan Spexd: 500 nm/min

Figure 3. SPECTROGRAM OF $0.05 \%$ METHYLENE BLUE PLUS IGTS8 CELLS 
WORK PLANNED FOR THE NEXT QUARTER

The work planned for the next quarter (August to October 1990) includes the following tasks:

- Continue the coal biodesulfurization experiments

- Develop and utilize techniques that will allow the genetic analysis of IGTS8

- Confirm the validity of a desulfurization assay based on the metabolism of methylene blue. 


\section{GLOSSARY}

Auxotroph - - A bacterial strain that contains a mutation in one or more genes involved in the synthesis of amino acids or vitamins. Such strains require that specific amino acids or vitamins are present in a bacterial growth medium to grow, because, unlike other bacteria (prototrophs), they lack the ability to synthesize a particular amino acid or vitamin.

Clone -- An identical copy. Clones can be entire organisms, such as identical twins, or molecular clones in which a single gene (or even a portion of a genel is copied/reproduced. Molecular cloning often involves obtaining a fragment of chromosomal DNA that encodes a desired gene and ligating/ fusing that DNA fragment to a plasmid. Because plasmids are capable of replication it will not only replicate itself, but also the newly inserted/cloned fragment of chromosomal DNA.

Conjugation -- Ir the self-directed transfer of plasmid DNA from one bacterium to another.

Gene -- Is a segment of DNA that is involved in producing a polypeptide chain.

Ligation -- Is the formation of a phosphodiester bond to link to adjacent bases separated by a nick in one strand of a double-stranded DNA molecule.

Phage -- (Bacteriophage) is a bacterial virus.

Plasmid - An extrachromosomal circular molecule of DNA capable of replication, often being present in multiple copies per cell. Plasmids are several orders of magnitude smaller than chromosomes and encode auxiliary functions. The chromosome encodes for all of the functions that allow a bacterial cell to reproduce and be identified as a particular species of microorganism; whereas, plasmids are not present in all bacteria and encode for specialized functions such as resistance to antibiotics. Plasmids can not only be inherited from one generation to the next, but can often also be transferred to other bacteria of the same or related species. This and the fact that plasmid DNA can be purified from bacterial cells with relative ease make plasmids useful tools in research involving bacterial genetics. The name of a plasmid always starts with a lower case letter p, for example pBR325.

Restriction Endonucleases (enzymes) -- Recognize specific short sequences of DNA and cleave the double-stranded molecule, most often yielding DNA fragments with short single-stranded ends. Various restriction enzymes are named by abbreviating the name of the species of origin followed by a nurber, that is, ECO Rl.

Transconjugant -- A bacterium that has acquired DNA by the process of conjugation.

Transduction -- Refers to the transfer of a gene from one bacterium to another by a phage. 
Transformant -- A bacterium that has acquired DNA by the process of transformation.

Transformation of Bacteria -- Describes the acquisition of new genetic markers by incorporation of added DNA.

Transposon -- Is a DNA sequences able to replicate and insert a copy of itself at a new location in the genome. Transposons are named with the prefix Tn, followed by a number, that is, Tn 917 .

Vector -- A general term referring to genetic elements (plasmids, viruses, transposons, and others) that contain or can be constructed to contain a DNA fragment of interest. Vectors are sometimes referred to as vehicles. 
APPENDIX .

Instantaneous Gene Transfer From Donor to Recipient Microorganisms Via Electroporation

A-1 


\title{
INSTANTANEOUS GENE TRANSFER FROM DONOR TO RECIPIENT MICROORGANISMS VIA ELECTROPORATION
}

\author{
John J. Kilbane II* \\ Barbara A. Bielaga \\ Insticute of Gas Technology \\ 3424 South State Street \\ Chicago, IL 60616
}

- Corresponding author. 


\begin{abstract}
Simplified electroporation methodologies have been developed that reliably yield transformants with only minutes of effort. Neither DNA purification, cells in specific phase of growth, cell washing, nor chilled cuvettes are required to obtain transformants. Electroporation can be used to transfer plasmid or chromosomal DNA directly from donor to recipient cells. This simplified direct method of electroporation has been demonstrated to work for both intra- as well as inter-species transformations using a variety of microorganisms.
\end{abstract}

A-5 


\section{INTRODUCTION}

Electroporation is the use of an electrical discharge to stimulate the transformation of eukaryotic or prokaryotic cells. Transformation of both plasmid and chromosomal DNA at frequencies equaling or exceeding those obtainable by conventional/alternative transformation procedures have been reported for a plethora of microorganisms and cell lines. $(1,3,4)$ Indeed, some microorganisms that are difficult or impossible to transform using conventional procedures have been successfully transformed using electroporation. $(5,6,7)$ However, the technique of electroporation is relatively new and much work remains as regards exploring the full range of applications of this technology and in methods development. Typically, the protocols employed in electroporation experiments are adapted from conventional transformation procedures and fail to take advantage of the unique characteristics of electroporation. Electroporation can not only be used to introduce DNA into a cell, it can also be used to get DNA out of cells. (3) Consequently, electroporation can be used to simultaneously get DNA out of one celi population and into another.

Research concerning the development or improvement of methodologies for electroporation have almost exclusively focused on achieving the maximum number of transformants per microgram of DNA. Methodologies optimized for the efficiency of transformation are indeed useful; however, there are many instances in genetic research in which obtaining only a single transformant will suffice. In those instances then, what is wanted is the simplest most rapid electroporation method that will reliably yield transformants, rather than conventional electroporation methodologies that are more complex and lengthy. The aim of the research reported here was to develop a simplified electroporation procedure for the transformation of various species of microorgansisms, and to compare the efficiencies of simplified procedures with those of conventional electroporation procedures. A preliminary report of procedures and data included here was presented at the annual American Society for Microbiology meeting in Anaheim, CA in May, 1990.

\section{MATERIALS AND METHODS}

The bacterial and plasmid species used in this study are listed in Table 1. Nutrient broth (Difco, Detroit, MI) was the growth medium used to 
obtain Iiquid cultures of all species. If solid medium was required, Nutrient Agar (Difco, Detroit, MI) was used for $\underline{E}$. coli and Rhodococcus species, but Pseudomonas Isolation Agar (Difco, Detroit, MI) was used for Pseudomonas aeruginosa. Antibiotics (Sigma, St. Louis, MO), when needed, were added at the following concentrations: ampicillin $100 \mathrm{ug} / \mathrm{ml}$, chloramphenicol $25 \mathrm{ug} / \mathrm{ml}$. rifampicin $250 \mathrm{ug} / \mathrm{ml}$, and tetracycline $25 \mathrm{ug} / \mathrm{ml}$. E. coli and p. aeruginosa cultures were incubated at 37 degrees Celsius. Rhodococcus cultures were incubated at 30 degrees Celsius. Bacterial growth in liquid culture was monitored turbidometrically using a Rlett-Sommerson colorimeter equipped with a green filter $(54)$ displaying approximately 100 Rlett units per $5 \times 10^{8}$ bacterial cells. Klett readings were used to determine if cells were in logarithmic or stationary phase: values of 300 units or greater defined cultures in stationary phase.

Cells in stationary or log phase were harvested from liquid culture by a ten-minute centrifugation at $10,000 \mathrm{rpm}$ in a Sorval SS34 rotor. The pellet was either resuspended in a small volume of distilled deionized water to yield concentrated cells ready for use in electroporation experiments, or the pellet was washed twice with distilled deionized water prior to electroporation. Both preparations produced a cell suspension concentrated 100-fold or more compared to the original culture volume (the final concentration was about $10^{10}$ cells per mll, although a few experiments were performed with more dilute cell preparations. Harvesting bacterial cultures from agar plates involved the selection of single colonies, multiple colonies, or streaks of bacterial growth with sterile toothpicks or an inoculating loop followed by suspension of the cells in a minimal volume of distilled deionized water to achieve a cell density of about $10^{8}$ cells per $\mathrm{ml}$.

The volume of cell culture used for electroporation was typically 100 microliters comprised of 50 microliters each of donor and recipient cells placed in cuvettes (either chilled or at room temperature) of $0.2 \mathrm{~cm}$ gap. Electroporation was performed with a Bio-Rad Gene Pulsem unit (model 1652076) equipped with a Bio-Rad pulse controller (model 1652098) adjusted to 2,500 volts and 25 microfarad capacitance and 200 ohms resistence yielding $12.500 \mathrm{~V} / \mathrm{cm}$ field strength. The time constants obtained under these conditions were typically about $5.5 \mathrm{miliseconds}$ for washe 3 cell suspensions and from 0.9 to $3.5 \mathrm{mill}$ iseconds for unwashed cell suspensions. Conditions other 
than these were tested; howeve=, the majority of experiments were performed using the standardized protocol described above.

DNA was obtained using the method of Birnboim et al. (2) and either used as is (crude lysate DNA) or was purified through two CsCl/ethidium bromide density gradients. Alternatively, DNA was obtained from cell preparations directly using electroporation. In this event three techniques were employed:

1). A suspension of plasmid-containing donor cells was pulsed, transferred to a microfuge tube then centrifuged at $14,000 \mathrm{rpm}$ fo: 30 seconds in an Eppendorf microfuge and the supernatant served as the source of DNA (this technique was designated as the pulse/spin/pulse method).

2). A suspension of plasmid-containing donor cells was pulsed, a suspension of recipient cells was added directly to the same electroporation cuvette and pulsed again, or,

3). A suspension containing a mixture of donor and recipient cells was pulsed.

A step-wise method for thr easiest method of electroporation appears in Figure 3 .

The concentration of DNA was measured either spectrophotometrically or by comparison of band intensities with standard DNA preparations on ethidium bromide stained agarose gels. The amount of DNA used in these experiments ranged from 0.01 to $0.25 \mathrm{micrograms.} \mathrm{After} \mathrm{electroporation,} \mathrm{the} \mathrm{cells} \mathrm{were}$ added to $4 \mathrm{mls}$ of nutrient broth, incubated for one hour at either 30 or 37 degrees Celsius and were then plated on selective agar.

\section{RESULTS AND DISCUSSION}

While electroporation has been reported to be useful in getting materials other than DNA into cells and in getting DNA (or other cellular constituents) out of cells $(3,8,9)$ it is not routinely used for purposes other than DNA transformation. We wanted to investigate using electroporacion as a means of purifying plasmid DNA directly from the cell. There are two easy ways to assay for the presence of DNA, visualizing DNA by agarose gel electrophoresis, and by transformation. CsCl purified DNA's were utilized as controls. A range of conditions were investigated to examine the potential of using electroporation to obtain DNA suitable for gel electrophoresis, and these are: bacterial growth phase, culture volume, cell concentration, cells 
washing procedures, chilled vs. room temperature cuvettes, voltage (6.25-12.5 $\mathrm{kV} / \mathrm{cm})$, capacitance $(0.25-25 \mathrm{uF})$, resistance (100-1000 ohms), multiple pulses (1-20), and cell outgrowth/recovery techniques.

The majority of experiments that were performed for the purpose of purifying DNA using electroporation involved BB101/pBR325; although, strains containing other plasmids were also examined. The relatively small size and high copy number of PBR325 was thought to make it a good candidate for use in DNA purification studies. Typically 30 to 100 microliter volumes of concentrated $\left(10^{10} \mathrm{cells} / \mathrm{ml}\right)$ cell solution were subjected to one or more electric pulses and either added directly to agarose gels or were microfuged at $14.000 \mathrm{rpm}$ for 30 seconds to remove cell debris and the supernatant was then analyzed on agaruse gels. In some cases, several aliquots of electrically pulsed cells were pooled, yielding a total volume of one to two milliliters. These pooled aliquots were subsequently microfuged, and DNA was precipitated from the supernatants using ethanol, resuspended, and analyzed on agarose gels.

The results of these attempts to purify pBR325 using electroporation are illustrated in Figure 1. While it is possible to obtain plasmid DNA using electroporation that can be visualized on agarose gels it is not an effective method for the purification of plasmid DNA. The yield of chromosomal DNA; however, compares favorably with other DNA purification methods, and yields a product that can be cleaved with restriction enzymes (data not shown). In general, the electroporation conditions that yield optimum results for the transformation of cells with plasmid DNA were also the best conditions for obtaining DNA that can be visualized on agarose gels, except that larger cell volumes (100 $\mathrm{kl}$ rather than $30 \mathrm{\mu l})$ yielded better results for purifying DNA, but had no significant impact on transformation frequencies.

In addition to assaying by agarose gel electrophoresis for the presence of plasmid and chromosomal DNA in electroporated cell suspensions a transformation/electroporation assay was also used. Procedures for optimal transformation by electroporation have been published in the literature. $1,3-6$ We began investigating an easier, quicker method of transformation and DNA purification utilizing these procedures. Initially. E. coli cells containing pBR325 were used as a source of pBR325 DNA that was obtained either by the alkaline lysis technique of Birnboim et al. (2) to yield a crude 
lysate, alkaline lysis followed by two CsCl/ethidium bromide density gradient purifications to yield highly purified DNA preparations, or by electroporation. The transformation efficiency of DNA solutions prepared by each of these methods was investigated using electroporation of $\underline{E}$. coli recipient cell cultures. The majority of experiments involved purified pBR325 DNA or cells containing pBR325; however, electroporation experiments were performed using all of the strains and plasmids listed in Table 1. To obtain DNA directly from cell preparations by electroporation three techniques described in the materials and methods section were employed. A range of conditions, as described above, were explored to obtain DNA via electroporation that is suitable for transformation. Standard electroporation conditions, as ulescribed in materials and methods, were used in transformation experiments employing DNA solutions. A comparison of the results obtained is illustrated in Figure 2. The highest transformation frequencies were obtained using CsClpurified DNA; huwever, DNA preparations obtained using electroporation and employed in the pulse/spin/pulse method performed quite well yielding only an order of magnitude decrease in the transformation frequency. Crude lysate DNA is the DNA obtained after Birnboim et al. (2) alkaline lysis and not purified any further. When E. Coli HBI01 log phase cells are the recipients of pBR 325 crude lysate DNA, the transformation efficiency is decreased by approximately $1 / 2$ as compared with purified DNA. The direct transfer of plasmid DNA from donor to recipient cells was also successful but at a greatly reduced frequency suggesting that the removal of cell debris from the DNA preparation has a profound effect on the subsequent transformation efficiency. The simultaneous pulsing of mixtures of donor and recipient cells generally yielded somewhat better results than sequential pulsing. Methods other than those used as standards have been tested. They include washed cell preps versus unwashed in both $\mathrm{ddH}_{2} \mathrm{O}$ and $108 \mathrm{glycerol}$ and the use of chilled cuvettes versus room temperature cuvettes. In general, neither the washing of cells in either $\mathrm{ddH}_{2} \mathrm{O}$ or 1.08 glycerol nor the condition of the cuvettes (chilled versus room temperature, have an appreciable effect on transformation frequency. However, washing cells does decrease the amount of arcing that occurs. We have found that transformation occurs even when there is arcing and that arcing can not be completely avoided even under the best conditions.

Knowing that enough DNA is released from cells after an electric pulse to be effectively used in transformation experiments this method of transferring 
DNA directly between 2 cell populations was investigated to examine the effects of plasmid size, bacterial strain, phase of growth, and the possibility of inter- as well as intra-species transformation. Confirming electroporation efficiencies using CsCl DNA served as controls for results with DNA obtained by electroporation. Plasmids varying in size from 2.7 kilobase pairs (Kb) to $50 \mathrm{~Kb}$ were electroporated into E. coli strain 6600 as well as other strains listed in Table 1. After testing the plasmids in Table 1. PBR325 and PCP13 were chosen for the rest of the work presented here. The fact that the number of transformants decreased as the plasmid size increased indicate that the electroporation efficiency is size dependent (Figure 2 ). The largest plasmid that will yield transformants in E. coli is unknown as even the largest plasmid employed here $(50 \mathrm{Rb})$ yielded transformants. This inverse relationship between plasmid size and transformation frequency is true of conventional transformation procedures as well as electroporation.

Growth phase and strain variation was also tested. CsCl purified PBR325 and PCPI3 were electroporated into E. coli strains HBI0l and C600. Cells were harvested from liquid culture at $l 0 g$ and stationary phase as well as from agar plates, washed and subjected to electroporation using purified DNA as well as DNA obtained by electroporation. In both strains, log phase cells transformed at the highest frequency. Log phase $c 600$ cells transform at 1.5 orders of magnitude greater frequency than HBl0l log phase cells illustrating the differences in transformation results even between cells from the same species. Stationary phase cells of c600 transform almost as well as log phase cells while in HBl0l, transformation occurs at 2 orders of magnitude less often with stationary phase cells as compared with log phase cells. Cells from agar plates, exhibit drastically reduced transformation frequencies. However, when only a few transformants are needed quickly the $t$ ime saved in cell preparation outweighs the fact that only a few transformants are obtained (see Figure 3 for protocol).

When studying transfer of DNA between two cell populations it is important to take into account the growth phase of both the donor and recipient, because the growth phase of the donor cells also effects the efficiency of transfer between two cell populations. The best donors come from stationary phase cultures. Donors directly off an agar plate, perform less well and log phase donors yield the lowest number of transformants (see Table 2 ). The

A-11 
question of whether this technique would work for interspecies as well as intraspecies transformation was addressed by comparing E. coli strains HBlOl, C600 and Pseudomonas aeruginosa strain PRS2015 using CsCl purified pCPl3 as well as the direct transfer of pCPl3 from donor to recipient cells. Electroporation conditions and growth phase of PRS2015 were as for the E. coli strains mentioned previously. Using CsCl purified pCPI3 DNA P. aeruginosa strain PRS2015 transforms at a frequency comparable to E. coli HBI0l, when using either $\log$ or stationary phase cells, whereas E. Coli strain C600 transforms at more than 2 orders of magnitude higher frequency comparing recipients from the same growth phases. Interestingly. PRS2015 cells from an agar plate transform at a higher frequency than either the log or stationary phase cells, and at a 1.5 order of magnitude higher than both E. coli strains ( Table 2).

Direct interspecies transfer of plasmid DNA between two cell populations was also investigated. E. CC.li HBl01/pCPl3 cells from an agar plate were used as donors and P. aeruginosa PRS2015 cells from log and stationary phase liquid cultures as well as cells from an agar plate were the recipients.

Interspecjes transfer of DNA from E. coli into p. aeruginosa was successful. Stationary phase recipients appear to be the most susceptible to transfer via electroporation and transiorn at a frequency of $4.9 \times 10^{2}$ transformants/ug DNA. Cells from an agar plate transform at a lower frequency and log phase cells transform poorly: $1.8 \times 10^{2}$ and 53.3 transformants/ug DNA respectively.

Additional interspecies transfer experiments have been performed. Plasmids from E. coli have successfully been electroporated into Salmonella typhimurium and Saccharomyces cerevisiae using simultaneous pulsing of donor and recipient cells. The frequencies of these transfers have not been accurately determined but appear to be consistent with the interspecies transformotion data reported here (J. Pfau, personal communication). Additionally the direct transfer of chromosomal markers from donor to recipient cells via electroporation has been detected in $\underline{E}$. colj by scoring for restoration/complementation of auxotrophy markers (data not shown).

In this report the use of simplified electroporation procedures for the transfer of plasmid and chromosomal DNA has been demonstrated; however, this technique should also be useful for the transfer of bacteriophage, RNA, or other cellular constituents from one cell populaticn to another. In fact, 
electroporation has already been used to introduce proteins and restriction enzymes into cells. $(8,9)$ Moreover, the technique might also have useful applications as a means of introducing dyes, inhibitors, antibiotics, ions, and other materials into cell populations.

\section{SUMMARY}

In conclusion, simplified electroporation methodologies have been developed that reliably yield transformants with only minutes of effort. Neither DNA purification, cells in specific phase of growth, cell washing, nor chilled cuvettes are required to obtain transformants. Electroporation can be used to transfer plasmid or chromosomal DNA directly from donor to recipient cells. This simplified direct method of electroporation has been demonstrated to work for both intra- as well as inter-species transformation using a variety of prokaryotic and eukaryotic microorganisms. 
1. Belliveau, B.H. and J.T. Trevors. 1989. "Transformation of Bacillus cereus Vegetative Cells by Electroporation." Applied and Environmental Microbiology, 55: 1649-1652.

2. Birnboim, H.C. and J. Doly.. 1979. "A Rapid Alkaline Extraction Procedure for Screening Recombinant Plasmid DNA." Nucleic Acids Research, 7: $1513-1523$.

3. Calvin, N.M. and P.C. Hanawalt. 1988. "High-Efficiency Transformation of Bacterial Cells by Electroporation." Journal of Bacteriology. 170: 2796-2801.

4. Dower, W.J., J.F. Miller, and C.W. Ragsdale. 1988. "High Efficiency Transformation of E. coli by High Voltage Electroporation." Nucleic Acids Research, 16: 6127-6145.

5. McIntyre, D.A. and S.K. Harlander. 1989. "Genetic Transformation of Intact Lactococcus lactis Subsp. lactis by High-Voltage Electroporation." Applied and Environmental Microbiology, 55: 604-610.

6. McIntyre, D.A. and S.K. Harlander. 1989. "Idproved Electroporation Efficiency of Intact Lactococcus lactis Subsp. lactis Cells Grown in Defined Media." Applied and Environmental-Microbiology, 55: 2621-2626.

7. Silke, D., G. Simons, and W.M. DeVos. 1989. "Plasmid Transformation by Electroporation of Leuconostoc paramesenteroides and It's Use in Molecular Cloning." Applied and Environmental Microbiology, 55:14831489 .

B. Winegar, R.A., J.W. Phillips, J.H. Youngbloom, and W.F. Morgan. 1989. "Cell Electroporation is a Highly Efficient Method for Introducing Restriction Endonucleases into Cells." Mutat. Res. 225: 49-53.

9. Winegar, R.A. and L.H. Lutze. 1990. "Introduction of Biologically Active Proteins into Viable cells by Electroporation." Focus 12: 34-37.

RPP/BioTeg/PP 
Table 1. BACTERIAL CULTURES AND PLASMIDS

Strain

\section{HBI0l rifampicin resistant}

C600 rifampicin resistant

EBI $01 /$ PBR 325

$\mathrm{HB} 101 / \mathrm{PCP} 13$

$\mathrm{HB} 101 / \mathrm{PBB} 4$

Pseudomonas aeruginosa PRS2015

Rhodococcus fasciens

Rhodococcus rhodochrous ATCC 19149

Rhodococcus rhodischrous ATCC 19067

Rhodococcus rhodochrous ATCC 13808

Rhodococcus rhudochrous IGTS 8

Rhodococcus globulus ATCC 19370

CsCl Purified Plasmids Used in Electroporation

$\underline{\text { Plasmid }}$

PAM 225

PBR 325

PCP 13

PGR71

pKH80

PYEJ1001

pUC8
Plasmid Size

$5.9 \mathrm{~Kb}$

$21.7 \mathrm{~Kb}$

$50.0 \mathrm{~Kb}$
Size, Kb

11.4

5.9

21.7

8.4

13.2

1.1

2.7

A-15 
Table 2. RESULTS OF SELECTED ELECTROPORATION EXPERIMENTS

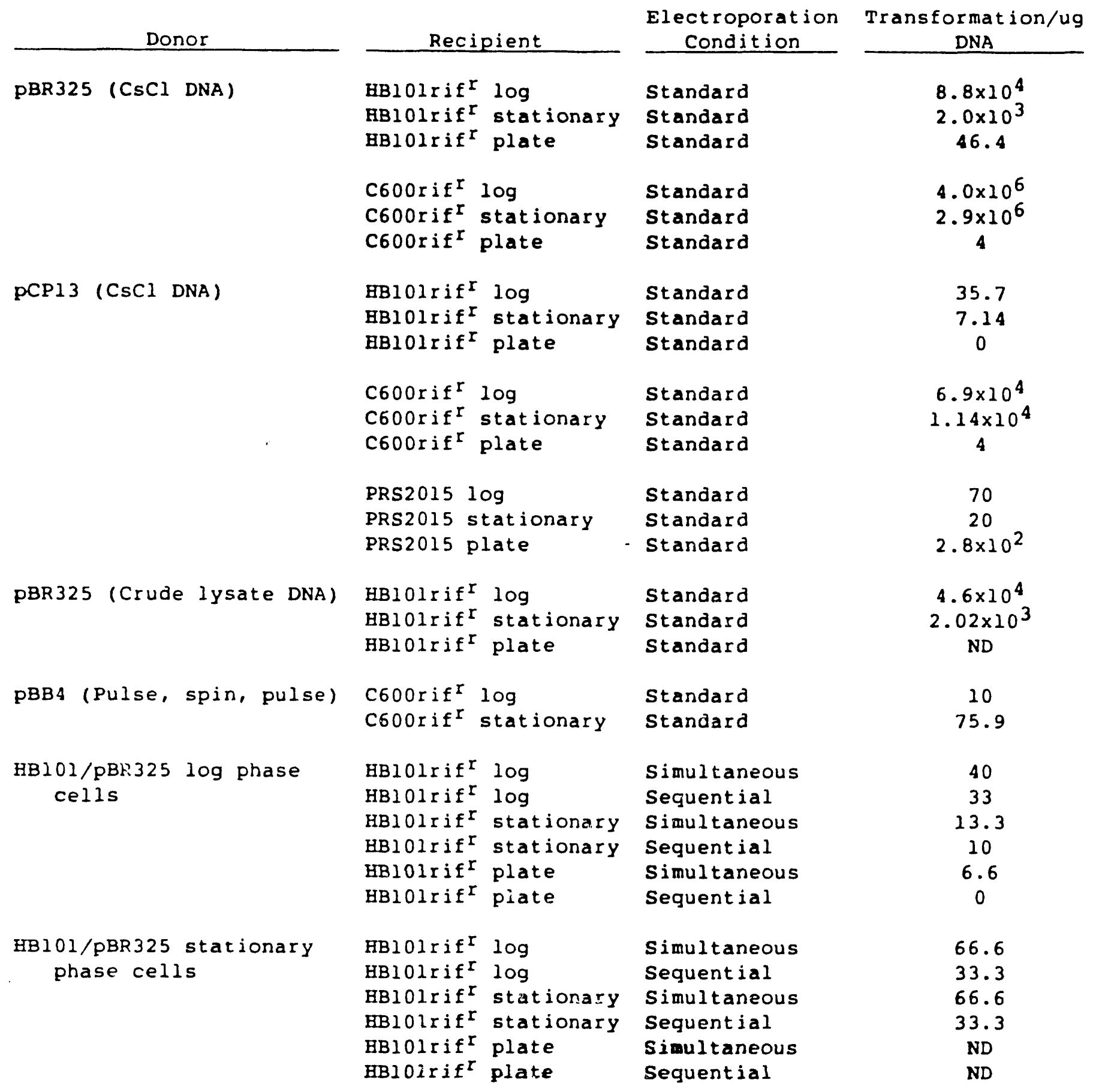


Table 2, Cont. RESULTS OF SELECTED ELECTROPORATION EXPERIMENTS

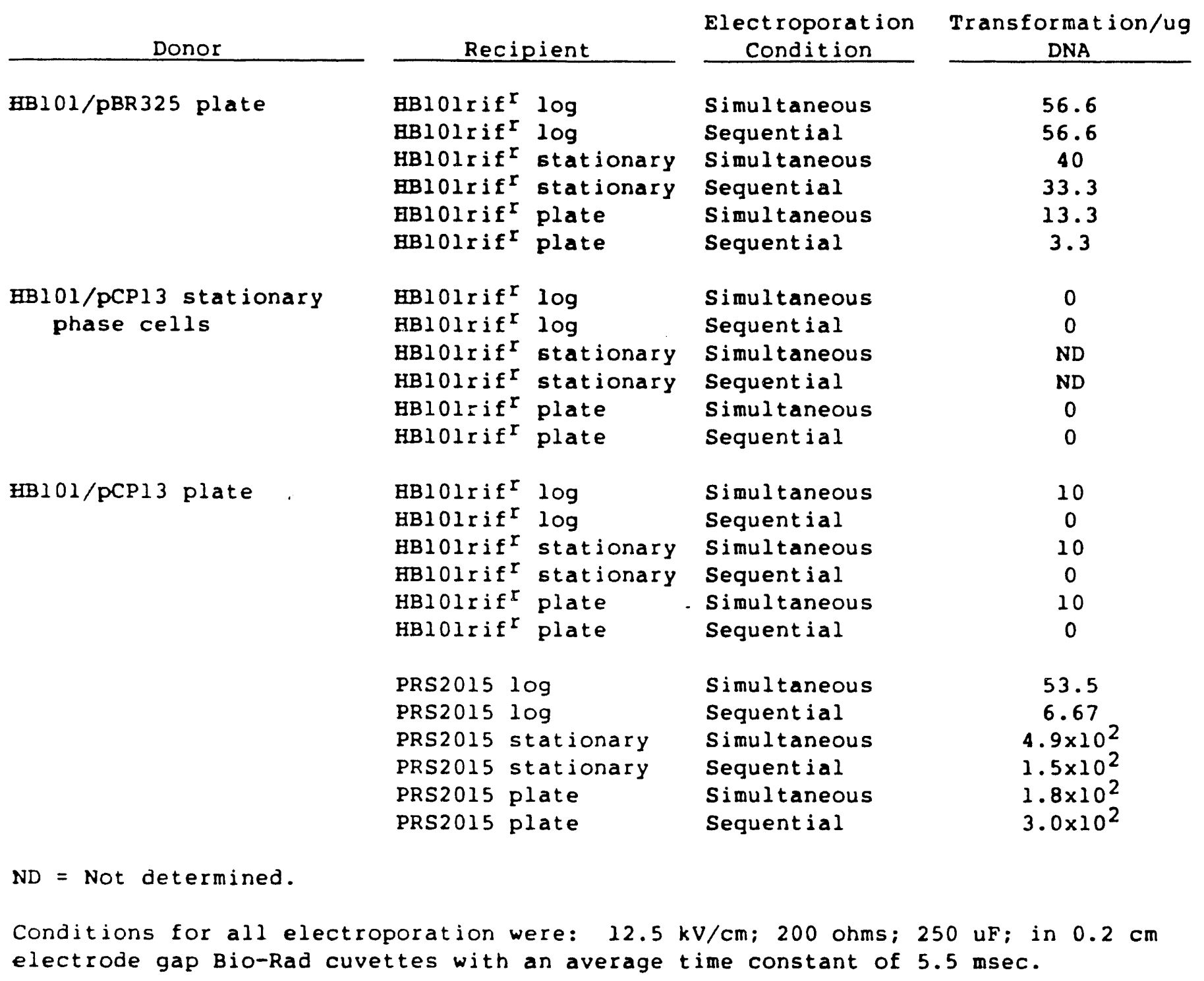

A-17

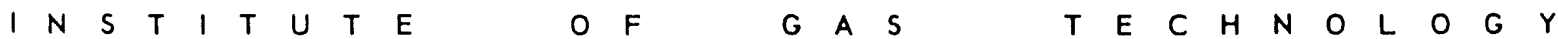




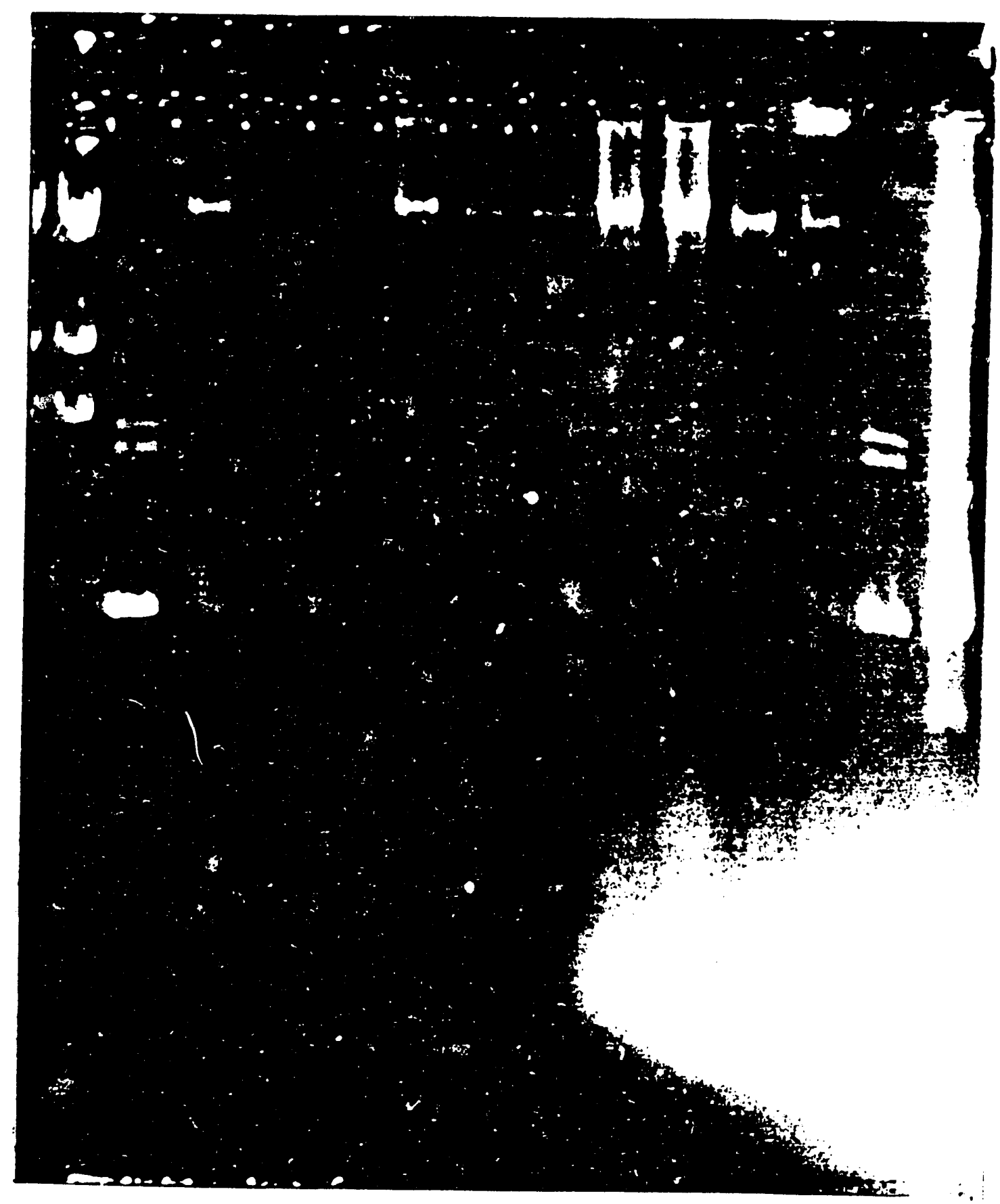

This ethidium bromide stained agarose gel depicts plasmid DNA removed from BB101/PBR325 via electroporation.

Lanes: (1) Hind III digested lambda DNA; (2) CsCl purified DNA from HBl01/pBR325; (3) $30 \mathrm{Hl}$ of $10^{10} \mathrm{cells} / \mathrm{ml}, 1 \mathrm{pu}$. se $(12,500 \mathrm{kV} / \mathrm{cm}, 200 \mathrm{ohm}, 25 \mathrm{HE})$; (4) $30 \mathrm{ul}$ of $10^{9}$ cells/ml, I pulse; (5) 30 ul of $5 \times 10^{8}$ cells/ml, 1 pulse; $(6-8)$ repeat of lanes 35, 5 pulses; (9) 100 ul of $10^{10}$ cells/ml, I pulse; $(10-12)$ repeat of lanes $3-5$ with 50 ul volumes, run without dilution; (13) CsCl purified DNA from HBI01/pBR325; (14)

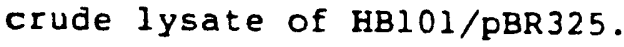

Figure 1. PURIFICATION OF DNA USING ELECTROPORATION 


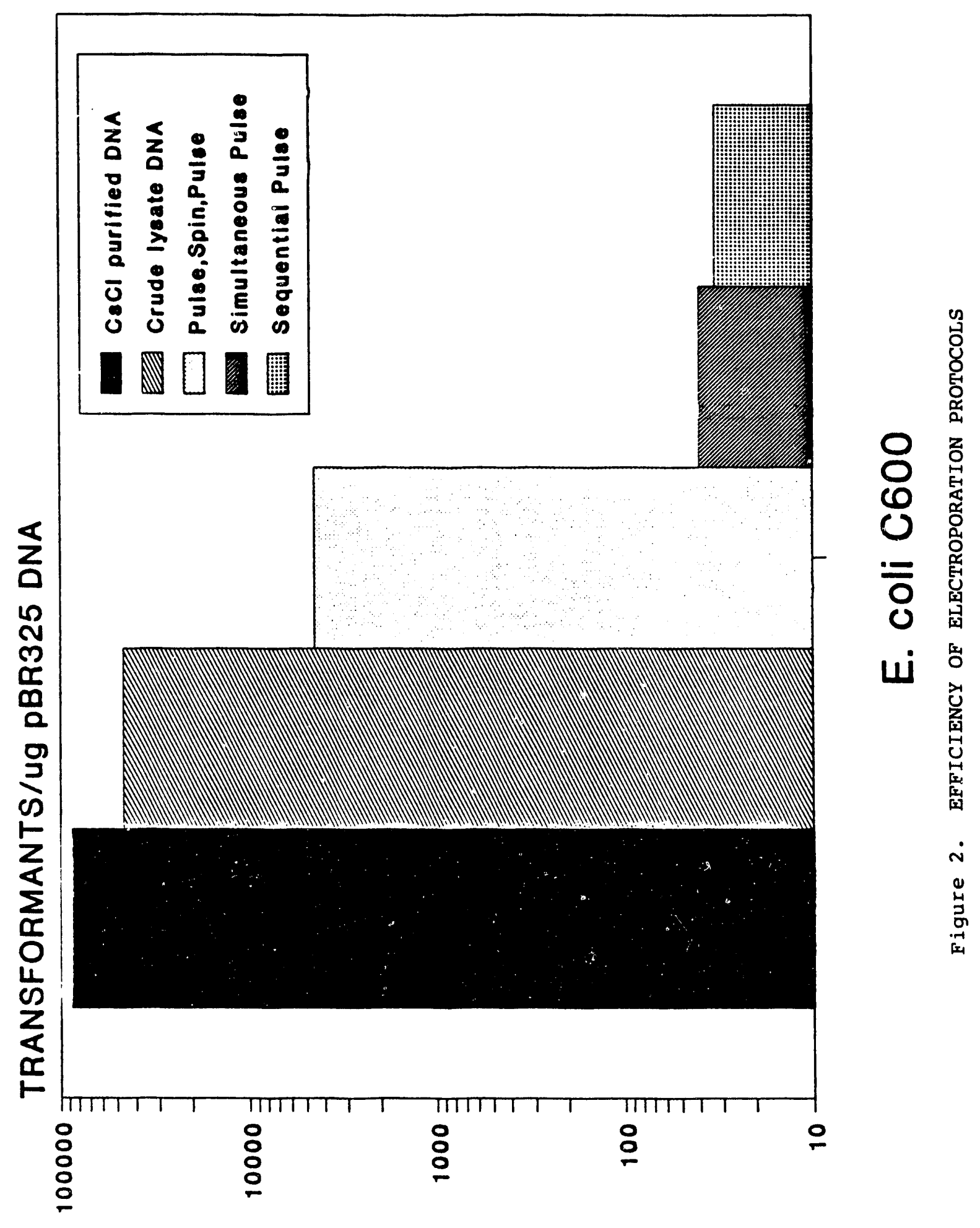

A-19 
1. Obtain 50 microliters of a washed cell suspension of $10^{10}$ donor cells per milliliter in sterile water (less dense solutions prepared from single colonies can be used).

2. Place the donor cell suspension in an electroporation cuvette and deliver an electrical discharge of $12.5 \mathrm{kV} / \mathrm{cm}$ at $25 \mu \mathrm{F}$ (multiple electrical pulses can be used).

3. Tzansfer the cell suspension to a sterile microfuge tube and spin at $14,000 \mathrm{rpm}$ for 30 seconds.

4. "Remove the supernatant to a second sterile microfuge tube that contains a sufficient amount of a washed recipient cell pellet to achieve a cell suspension of $10^{10}$ cells/ml.

5. Transfer the cell suspension to a fresh electroporation cuvette and deliver an electrical discharge of $12,500 \mathrm{kV} / \mathrm{cm}$ at $25 \mu \mathrm{F}$ (or at conditions more appropriate for the particular recipient cell culture being used).

6. Transfer the cell suspension to a test tube containing $5 \mathrm{mls}$ of Luria broth and incubate for 1 hour at $37^{\circ} \mathrm{C}$ (or other appropriate temperature).

7. Plate various dilutions of the cell suspension on selective agar, incubate overnight at $37^{\circ} \mathrm{C}$ to obtain transformants.

This procedure assumes that the cell cultures used possess genetic markers such that transconjugants car be uniquely selected from a mixture of donor and recipient cells. If this is not the case, the supernatant obtained in step 3 can be passed through a 0.4 micron filter to obtain a sterile cell-free DNA suspension that can be employed with any recipient strain in step 4. However, the filter sterilization of volumes of $50 \mathrm{\mu l}$ or less requires sample dilution (with sterile water) and a subsequent decrease in the number of transformants obtained.

donor (HB101/pBR325)

1.

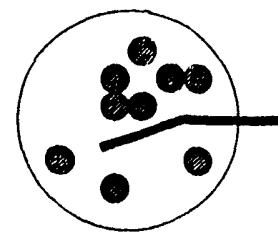

single colony selected

from each plate

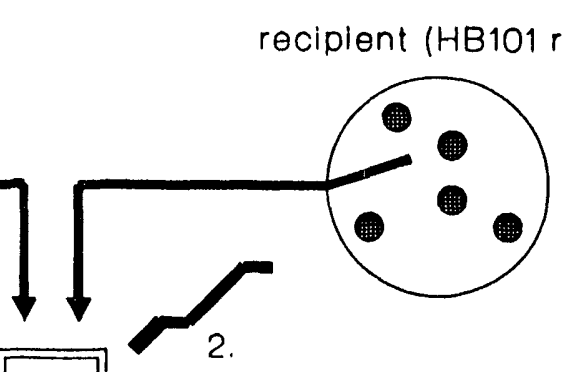

voltage opens pores in bacterial membranes allowing DNA to leave donor and enter recipient
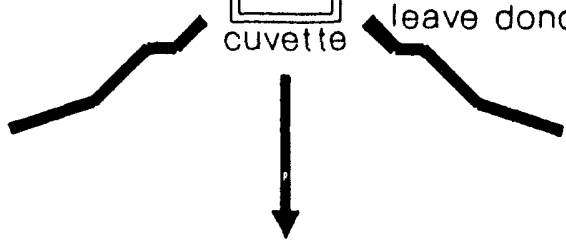

3.

transformants grow on selective agar

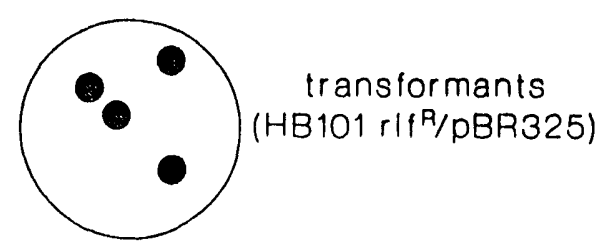

Figure 3. RECOMMENDED PROCEDURE FOR OBTAINING THE HIGHEST FREQUENCY OF TRANSFORMANTS IN THE MOST CONVENIENT FASHION 

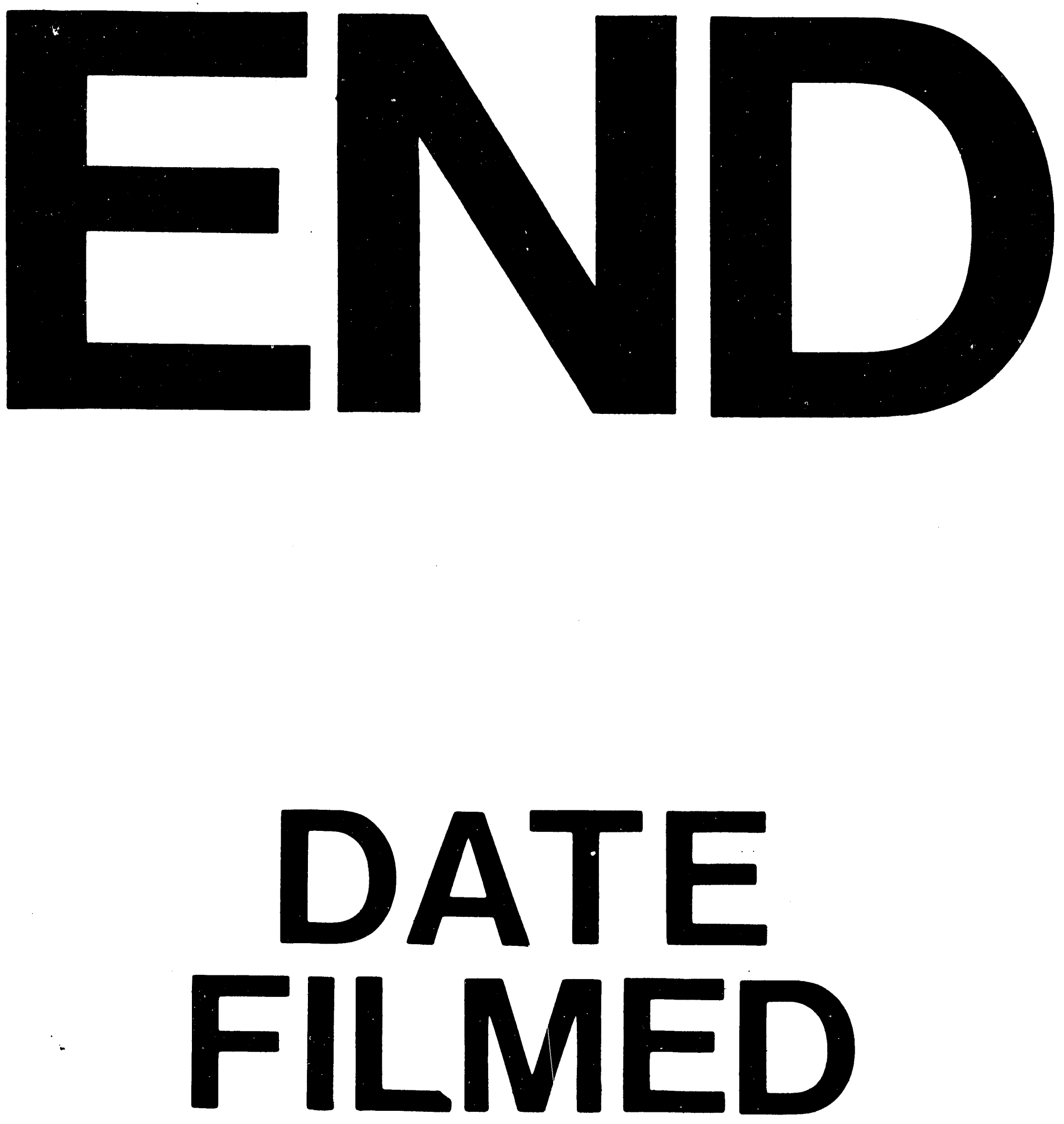

1

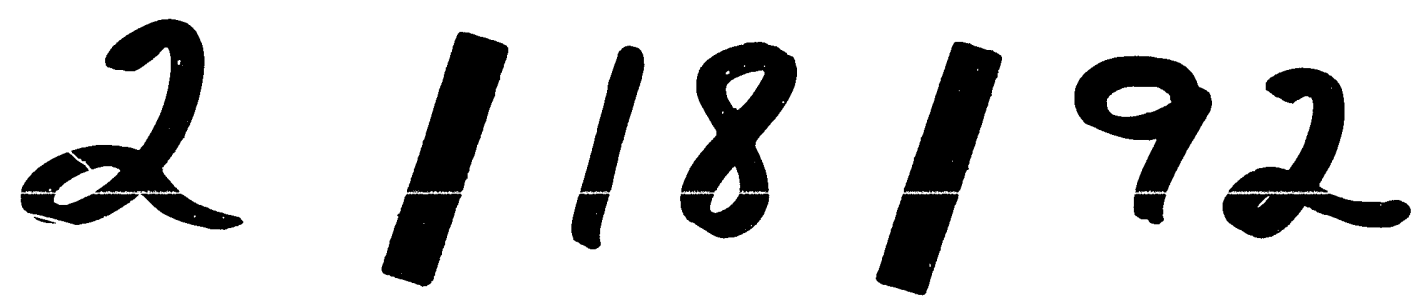


